\title{
Generation of Photoionized Plasmas in the Laboratory: Analogues to Astrophysical Sources
}

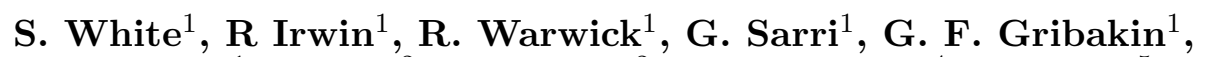 \\ F. P. Keenan ${ }^{1}$, E. Hill ${ }^{2}$, S. J. Rose ${ }^{2}$, G. J. Ferland ${ }^{4}$, F. Wang ${ }^{5}$, G. \\ Zhao $^{6}$, B. Han $^{6}$ and D. Riley ${ }^{1}$ \\ ${ }^{1}$ Centre for Plasma Physics, Queen's University Belfast, Antrim, United Kingdom \\ ${ }^{2}$ Imperial College, Faculty of Natural Sciences, London, United Kingdom \\ ${ }^{3}$ Clarendon Laboratory, University of Oxford, Parks Road, Oxford, United Kingdom \\ ${ }^{4}$ Department of Physics and Astronomy, University of Kentucky, Lexington, Kentucky, USA \\ ${ }^{5}$ Key Laboratory of Optical Astronomy, National Astronomical Observatories, Chinese \\ Academy of Sciences, Beijing, China \\ ${ }^{6}$ Department of Astronomy, Beijing Normal University, Beijing, China
}

\begin{abstract}
Implementation of a novel experimental approach using a bright source of narrowband x-ray emission has enabled the production of a photoionized argon plasma of relevance to astrophysical modelling codes such as Cloudy. We present results showing that the photoionization parameter $\xi=4 \pi F / n_{e}$ generated using the VULCAN laser was $\approx 50 \mathrm{erg} \mathrm{cm} \mathrm{s}^{-1}$, higher than those obtained previously with more powerful facilities. Comparison of our argon emissionline spectra in the $4.15-4.25 \AA$ range at varying initial gas pressures with predictions from the Cloudy code and a simple time-dependent code are also presented. Finally we briefly discuss how this proof-of-principle experiment may be scaled to larger facilities such as ORION to produce the closest laboratory analogue to a photoionized plasma.
\end{abstract}

Keywords. Plasma, Photoionization, Lab Astro

\section{Introduction}

Photoionized plasmas occur in accretion-powered compact objects such as x-ray binaries and the cores of active galaxies. As material accretes onto the compact object, gravitational potential energy is converted into an intense x-ray radiation field. This subsequently photoionizes the surrounding material generating plasma where ionisation is dominated by radiative processes compared to collisional ones. The degree of dominance can be characterised by the photoionization parameter $\xi=4 \pi F / N_{e}$, where $F$ is the flux and $n_{e}$ is the electron density, which can reach $\sim 1000$ erg $\mathrm{cm} \mathrm{s}^{-1}$.

The production of such a plasma in the laboratory would thus be of great interest to the astrophysical community as it would allow benchmarking of plasmas modelling codes such as Cloudy. However, since laboratory densities are much larger than the astrophysical case, the intensity of the radiation field must also scale accordingly, placing large constraints on any experiment. Previous experiments on major facilities have produced a quasi-blackbody x-ray sources to drive photoionization, such as Loisel et al. (2017) and Foord et al. (2004) at the Z machine at Sandia National Laboratory and Fujioka et al. (2009) at the GEKKO-XII laser facility. These experiments have achieved $\xi \approx 6$ $25 \mathrm{erg} \mathrm{cm} \mathrm{s}^{-1}$ at $\mathrm{n}_{e} \approx 10^{19}-10^{20} \mathrm{~cm}^{-3}$.

Here we present results of a proof-of-concept experiment that used a novel approach proposed by Hill and Rose (2011) that replaces the quasi-blackbody source with an intense relatively narrow-band line radiation source. This provides the necessary high 
x-ray flux at keV energies such that photoionization dominates the plasma dynamics but drastically lessens the demands required on the facility to generate such a source. This approach is also inherently scalable to larger facilities to achieve values of $\xi>$ $100 \mathrm{erg} \mathrm{cm} \mathrm{s}^{-1}$.

\section{Experiment}

The experiment was carried out at Target Area West (TAW) in the VULCAN laser facility at the Rutherford Appleton Laboratory. Figure 1 shows a schematic layout for our experiment. The Ar sample was contained in an $\mathrm{Al}$ gas cell, with a re-entrant Al plug on the 'east' face and $20 \mu \mathrm{m}$ thick kapton windows (shown as yellow) on the remaining faces. A hypodermic needle inserted at the base of the cell acted as a fill line and mounting post. The Ar could be filled in-situ under vacuum to precise pressures in the range of 50-500 mbar. At the centre of the re-entrant plug was a $0.5 \mathrm{~mm}$ diameter hole in a $0.5 \mathrm{~mm}$ thick base. Placed over this hole, on the exterior surface was an $18.8 \mu \mathrm{m}$ thick polypropylene layer coated with $218 \mathrm{~nm}$ of Sn. Incident upon this Sn layer were six frequency doubled $(\lambda=527 \mathrm{~nm}$ ) beams all focused on the centre of the $0.5 \mathrm{~mm}$ aperture to an overlapped focal spot of $400 \mu \mathrm{m}$ diameter. In total about $850 \mathrm{~J}$ of laser energy in a 1.5 ns flat top pulse was delivered onto target.

This produced a source of Sn L-shell radiation between $3.4-4.3 \mathrm{keV}$, whose emission was partially collimated by the $0.5 \mathrm{~mm}$ aperture in the re-entrant plug, and which then photoionized the Ar sample. The subsequent fluorescent $\mathrm{K} \alpha$ and $\mathrm{K} \beta$ emission from Ar was recorded through the 'south' window by a spherically curved mica crystal spectrometer. This emission was spatially resolved along the axis normal to the Sn by setting the spectrometers dispersion plane vertically. A flat HOPG crystal coupled to an x-ray streak camera (XRSC) recorded the temporal profile of the Sn x-ray emission. Two flat Si (111) crystals coupled to x-ray CCDs, looked either directly at the laser irradiated side of the target, or (on the 'west' side) at emission through the polypropylene layer. Another flat TAP crystal spectrometer was fielded on some shots to estimate the contribution of lower energy, $(\approx 1.5 \mathrm{keV})$ x-rays. Finally a pinhole camera and x-ray CCD imaged the spatial profile of the $\mathrm{x}$-ray source.

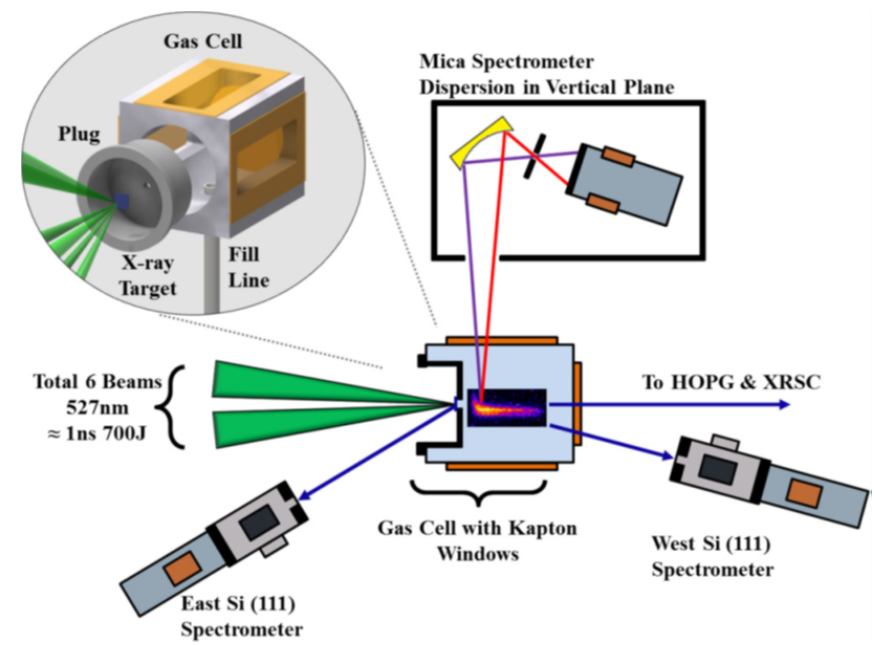

Figure 1. Schematic of the Experiment. 


\section{Results}

Figure 2 shows a typical image from our Mica spectrometer. Clearly visible is strong emission from both $\mathrm{Ar} \mathrm{K} \alpha$ and $\mathrm{K} \beta$ from about $0.5 \mathrm{~mm}$ to $5.5 \mathrm{~mm}$ away from the Sn target. Also visible on the other side of the target is weak emission, most likely occurring at multiple orders and coming from the Sn ablation plume later in time. Between these features we see the shadow region cast by the gas cell itself. The lower image in Figure 2 shows lineouts across the $\mathrm{K} \alpha$ feature taken about 0.5-0.7 mm from the $\mathrm{Sn}$ target, i.e. as close to the Sn source as we can see. Red squares at the top show the calculated spectral positions for the $\operatorname{Ar} \mathrm{K} \alpha$ ionisation stages. For pressures between 150 and $1000 \mathrm{mbar}$ we obtain similar levels of ionisation with the average ionisation, $Z^{*} \approx 4$ while we see a significant drop in ionisation for the 50 mbar to $Z^{*} \approx 3$. Since we can precisely vary the initial Ar density, and on the timescales of our experiment we can neglect hydrodynamic motion, we estimate $\mathrm{n}_{e} \sim 3.6 \times 10^{19} \mathrm{~cm}^{-3}$ for the 50 mbar case.
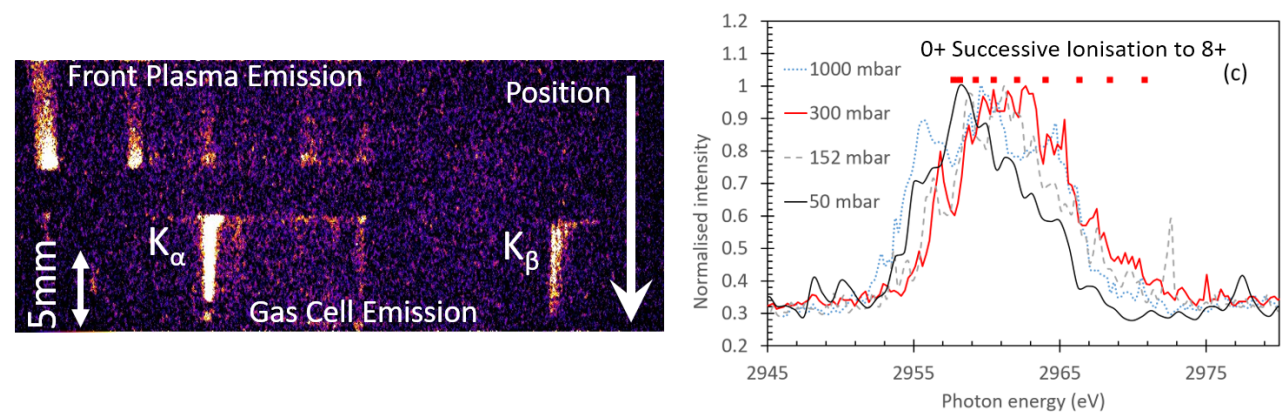

Figure 2. Left: Typical Image from our Mica Spectrometer. Right: Lineouts at four different pressures $\approx 0.5-0.7 \mathrm{~mm}$ from the $\mathrm{Sn}$. Red arrow inset on CCD image shows approximate direction and position where these lineouts were taken. Colour Online
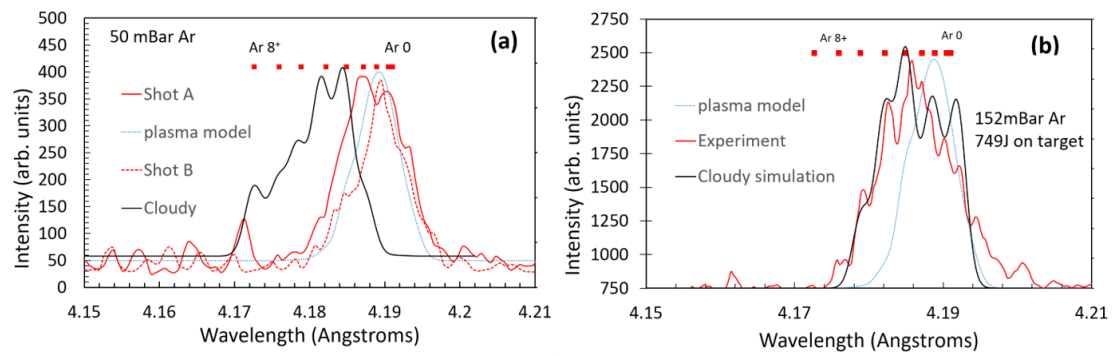

Figure 3. Comparison to Cloudy and our simple plasma model at two different pressures.

Analysis of the Sn spectrum transmitted through the polypropylene showed that $\approx 2.0 \pm 0.5 \%$ of the incident laser energy is converted to L-shell x-rays on a shot. From our TAP spectrometer and scaling from previous work on Pd (Kettle et al. 2015) we can estimate the contribution of lower energy x-rays passing through the $\mathrm{CH}$ layer and into the Ar. This gives an effective blackbody temperature of $175 \pm 10 \mathrm{eV}$. Results from the XRSC showed that the L-shell emission had a typical duration of about 500 ps FWHM. To account for the spatial profile of the x-ray source we fitted a super-Gaussian to pinhole camera data and assumed a $\cos ^{1 / 2} \theta$ distribution in x-ray emission. Based on the geometry we were able to estimate the average flux as $\sim 10^{19} \mathrm{erg} \mathrm{cm}^{-2} \mathrm{~s}^{-1}$. Thus for the 50 mbar case we have $\xi \approx 50 \mathrm{erg} \mathrm{cm} \mathrm{s}^{-1}$. 
In Figure 3 (a) we compare two shots at 50 mbar pressure to both Cloudy (Ferland et al 2017) and a simple time dependent model, while Figure 3 (b) shows a similar comparison for a 150 mbar shot. Cloudy is good at describing the higher pressure case, while is clearly in disagreement at 50 mbar. The degree to which Cloudy agrees may already be surprising as it is a steady-state model, and an analysis of our experiment shows we do not reach steady-state conditions. We have also shown that non-local energy transport via thermal electrons produced near the Sn x-ray source can have an important effect on the target conditions. A detailed discussion on these issues is beyond the scope of this article but may be found in White et al. (2018).
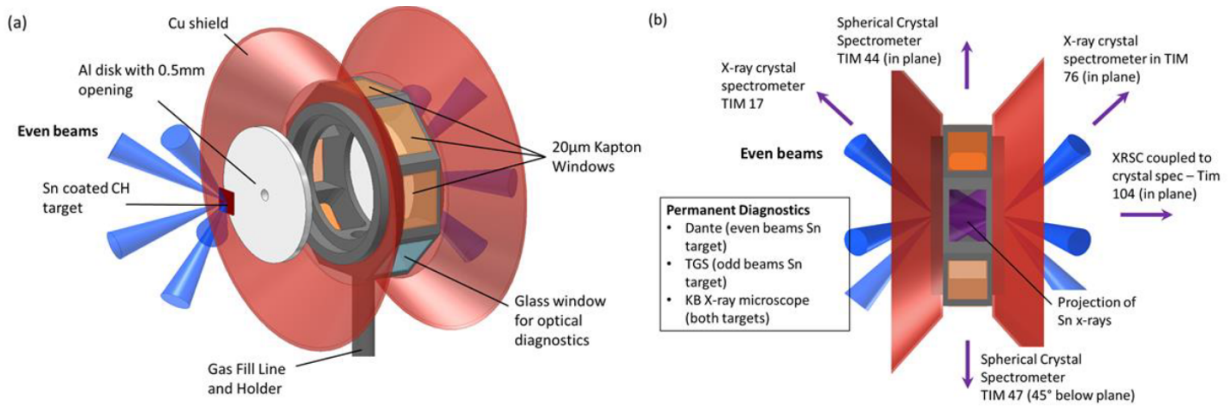

Figure 4. Proposed layout for ORION. The target is irradiated on both sides by 5 beams at $\lambda=351 \mathrm{~nm}$. Also shown are the lines-of-sight for the permanent diagnostics present at ORION.

\section{Scaling to Larger Facilities}

The design of our experiment easily scales to larger facilities, and here we discuss a planned experiment on ORION. We will employ an advanced target design that will facilitate simultaneous double-sided irradiation. This allows an increased flux at the centre of the Ar sample as well as more uniform conditions. At ORION we can also exploit the fact that the drive beams are at $3 \omega$ and should be much more efficient at converting to Sn x-rays. This can lead to as much as a six-fold increase in x-ray flux and peak values of $\xi>100 \mathrm{erg} \mathrm{cm} \mathrm{s}^{-1}$ at $\approx 0.5 \mathrm{~mm}$ from the $\mathrm{Sn}$ x-ray source. Figure 4 shows the proposed experimental layout for ORION. We can also take advantage of a comprehensive suite of diagnostics to produce a very well diagnosed photoionized plasma.

\section{Conclusions}

We have successively conducted a proof-of-principle experiment to produce photoionized plasmas using VULCAN. By employing a novel technique using the relative efficiency of a narrowband x-ray line emission source, we have achieved high values of $\xi$ using a relatively small laser facility. We have also shown that we can generate high quality fluorescent emission spectra from Ar for comparison to models used in the astrophysical community. Finally we discussed extending this technique to large laser facilities.

\section{References}

Ferland et al. 2017, Rev. Mex. Astron. Astrofis., 53, 385

Foord et al. 2004, Phys. Rev. Lett., 93, 055002

Fujioka et al. 2009, Nat. Phys., 5, 821

Hill and Rose 2011, High Energy Density Physics, 7, 377 
Kettle et al. 2015, J. Phys. B, 48, 224002

Loisel et al. 2017, Phys. Rev. Lett., 119, 075001

White et al. 2018, Phys. Rev. E, 97, 063203 